\title{
Essay 2: \\ Equilibrium Market Segmentation for Targeted Pricing Based on Customer Characteristics
}

\author{
Ke-Wei Huang ${ }^{1}$ \\ Leonard N. Stern School of Business, New York University \\ 44 West 4th Street, New York, NY 10012 \\ khuang0@stern.nyu.edu, \\ March 20, 2007
}

\begin{abstract}
Recent advances in information technologies create numerous opportunities for retailers to turn customer information into additional profits by targeted pricing: charging different prices to different market segments based on customer demographic variables. However, rigorous theoretical analysis regarding what is the profit-maximizing set of variables remains scarce. This study develops a game-theoretic model to investigate this question. Results of this model suggest that a monopoly seller should use pricing variables with high explanatory power of its demand: i.e., variables with large demand coefficient or high variance. In the duopoly case, this model suggests that the value of price discrimination is the same as that in the monopoly case when only one firm uses that pricing variable. When two symmetric firms simultaneously use a pricing variable, the value of price discrimination may be higher or lower. When two firms sell substitutes and the demand is affected by that variable in the same direction, VOPD is higher. In contrast, when the demand is affected by that variable in the opposite direction, VOPD is lower. When firms sell complements, these effects are reversed. This model is applied to horizontal differentiation and vertical differentiation to explain the puzzle that in practice similar products are often priced based on different variables.
\end{abstract}

Keywords: Coupons, Database Marketing, E-Commerce, Personalized Marketing, Price Discrimination, Pricing Research, Promotion, Segmentation, Targeting.

\footnotetext{
${ }^{1}$ This paper is a chapter of my dissertation at New York University. I would like to thank my advisor, Roy Radner, for his encouragements and numerous helpful suggestions. I would also like to thank the following people for their comments on the earlier drafts of this paper: Lorin Hitt, Debabrata Dey, DJ Wu, Anindya Ghose, Arun Sundararajan, Joel Steckel, William Greene, Foster Provost, Ying-Ju Chen, seminar participants at Workshop on Information Systems and Economics 2006, Stern School of Business, University of Connecticut, Georgia Tech., and Wharton School of Business. This project has been supported by Taiwan Merit Scholarship(TMS-094-1-A-043). The usual disclaimer applies.
} 


\section{Introduction}

In the Internet age, retailers are privileged in knowing what products customers buy, at what price, and the demographic variables of each customers. The question is how retailers should use this information. Recent advances in information technologies create numerous opportunities to turn customer information into additional profits. For example, business intelligence software can rank customers, and identify and prioritize the best segments based on customer information. Firms began by including targeted messaging on webpages, emails, and catalogues with a customer's preferred products and special offers. In a recent Yankee Group survey, 48 percent of the 456 respondents said they currently use technology to manage pricing. Meanwhile, 25 percent plan to buy technology in the next 12 months to provide customer-specific pricing electronically. This is because pricing software generates an impressive ROI, typically 5 to 19 percent profit improvement (source: www.cio.com). However, rigorous theoretical analysis regarding what is the optimal set of demographic variables sellers should use for price discrimination and/or market segmentation remains scarce. When it is unclear which set of demographic variables the sellers should use to create market segments, efforts to reach customers and improve customer profitability will fall flat.

Behind the fancy names such as targeted pricing, targeted couponing, and promotion management is a long-existing economic concept: price discrimination. The selection of pricing variables is crucial for any price discrimination strategies. Surprisingly, existing literature focuses only the price points but not the variables on which the prices based. Paradoxical phenomenon exists even in the most conventional, daily price discrimination scenarios. For example, stores around a university all have the following options of price discrimination strategies: (1) no discounts at all; (2) discounts to only students (with student ID); (3) discounts to everyone who works in that university (with university ID); (4) issuing coupons to passengers on the street; (5) coupons to returning/loyal customers; (6) restaurants or stores may charge higher prices to university staff because of convenience. All of these strategies coexist around any university in the United States. However, to the best of my knowledge, a thorough analysis of this phenomenon has not been provided in the literature.

A high-tech version of this story occurs in the enterprise software industry: similar products 
or even the same product in different generations are priced based on different variables. For example, most of the software products are charged based on the (concurrent) number of users or computers. At the same time, IBM mainframes have been priced based on the horsepower of CPUs for years. Sun Microsystems' Java Enterprise System has been priced by the size of the purchasing company's staff. Databases have been priced by the number of CPUs. Once Oracle, the most powerful database vendor, experimented for two years with licensing based on the total processing power of CPUs, but abandoned the new pricing scheme after Oracle 9i. Since the variable cost of these software products is zero, these practices are price discrimination examples in essence. Without the understanding of the underlying trade-offs, even a dominant, hundredbillion dollar company, such as Oracle, may incur huge loss due to failed pricing experiment.

The pricing variables selection problem has much wider applicability than these two examples. Online brokers, such as eTrade, can charge customers based on the number or dollar amount of transactions. eBay charges auction sellers by a complicated function of initial and final prices. Rental cars can be charged based on actual mileage, prepaid mileage, or the length of rental time. The same seat of the same flight may be sold from $\$ 200$ to $\$ 1000$ based on a variety of factors. Digitized books/music/movies can be sold based on the number of concurrent users (or computers), the number of times that the file can be used (usage), or the length of the ownership. Online DVD rental firms Netflix and Blockbuster charge users based on the number of DVD rented at the same time or the total number rented in a month.

Motivated by burgeoning applications, this study attempts to answer the following research questions: (1) How should firm(s) choose pricing variables in a monopoly (or duopoly)? How does competition affect the variables selection problem? (2) Under what conditions will firms benefit from pricing based on the same variable? In the database example, since the most powerful vendor cannot unilaterally changed the pricing variable, it is possible that pricing based on the same variable is an equilibrium in that case. The present study confirms this conjecture. At the first sight, this fact seems to contradict the intuition that firms can relax price competition by using different variables. This study will address these issues and provide the conditions under which each pricing strategy profile can be an equilibrium. 


\subsection{Overview of Results}

This paper investigates this problem by using a third-degree price discrimination model. Examples in the introduction are typical third-degree price discrimination. ${ }^{2}$ This study models consumer demand using a general linear demand system, which encompasses models with horizontal or vertical differentiation. Specifically, the demand of each firm is assumed to be a linear combination of pricing variables and prices. No distributional assumption is imposed on the pricing variables except assuming zero correlation between any two variables. The sequence of the game is as follows: risk-neutral firms first select pricing variables simultaneously and then choose pricing functions simultaneously second.

In the benchmarking monopoly case, this model suggests that the optimal pricing plan is a linear function of the pricing variables (a two-part tariff). The additional value of using a pricing variable depends only on three factors: (1) the monopoly power (2) the variance of that variable (3) the coefficient of that variable in the demand function. Interestingly, this value of price discrimination (VOPD) does not depend on the mean of each variable. Therefore, it is optimal for the monopoly seller to use pricing variables with high explanatory power of its own demand: i.e., variables with large coefficients or high variance. In other words, this "variance reduction" criterion is similar to selecting independent variables for a linear regression on firm demands. It is worth noting that, unlike linear regression, the goal of this model is to maximize expected firm profit rather than to minimize mean-squared error. Nevertheless, the optimal rule of selecting variables is the same. This model also suggests a simple index for estimating the percentage of revenue improvement. Without this index, marketing managers can estimate the revenue gain only via expensive pricing experiments.

\footnotetext{
${ }^{2}$ Second-degree price discrimination occurs when prices differ depending on the number of units of the good bought, but not across consumers (Varian (1992)). Third-degree price discrimination means that different purchasers are charged different prices, but each purchaser pays a constant amount for each unit of the good bought (Varian (1992)).

In some of the motivational examples, buyers can change their pricing variables, which is a seconddegree price discrimination problem. For example, firms can change the number of employees to save software expenses. However, few firms adopt this strategy in practice. In the extreme, even student status is "adjustable" by getting a fake ID or registering for a class. But, few people will act this way to get student discounts. Hence, results of this papers still apply well in examples when buyers do not change their characteristics because of the pricing plan.
} 
In the duopoly case, this model suggest that the VOPD is the same as that in the monopoly case when only one firm uses that pricing variable. When two symmetric firms use a pricing variable at the same time, the VOPD may be higher or lower. When two firms sell substitutes and the demand is affected by that variable in the same direction, VOPD is higher. The reason is that price discrimination based on the same pricing variable can better coordinate firms' pricing strategies to relax competition. In contrast, when the demand is affected by that variable in the opposite direction, VOPD is lower. The intuition is similar to the literature in targeted pricing, in which price discrimination will trigger price war in each firm's strong/loyal market segments, leading to lower equilibrium VOPD. When firms sell complements, these effects are reversed.

This paper provides two stylized applications to horizontal differentiation and vertical differentiation. In the first case, it is shown that pricing based on the same variable will damage the VOPD. In the vertical differentiation case, this study shows that the low quality firm has stronger incentive to use variables that affect only the willingness-to-pay of customers whereas the high quality firm has stronger incentive to use variables that affect only the quality preference of customers. In both cases, this model suggests that multiple Nash equilibria is the norm rather than the exception, providing one convincing explanation of why similar products are priced based on different variables in practice.

\subsection{Relevant Literature}

This paper adds to the existing literature in several important aspects. First, to the best of my knowledge, this study is the first to investigate the pricing variables selection problem, which is crucial to the success of a fundamental marketing problem: pricing. As discussed in the introduction, in the digital age, the variable costs of more and more products are becoming

negilible, leading to more new pricing plans and wider selection of pricing variables. At the same time, industrial environment becomes more volatile, the new pricing plans become more short-lived and it is also more costly to find optimal pricing plans by market experiments. A theoretical model can greatly save the time and efforts of practitioners to find the new pricing plans that use most profitable pricing variables.

Second, the present paper complements the existing literature that studies the value of de- 
mand information in a duopoly model (Bertrand-Nash pricing game). When firms use a variable, they can charge different prices in different market segments, which is similar to charging different prices based on signals of market demand conditions. It has been shown in the seminal work of Vives (1984) that in a duopoly model where firms have private information about an uncertain linear demand, if the goods are substitutes, to share information is a dominant strategy for each firm in Bertrand competition with known cost structures. If the goods are complements, the result is reversed. Along this line, numerous papers have investigated the conditions under which oligopolists have an incentive to share private information about a stochastic demand or stochastic costs (e.g., Sakai (1985), Gal-Or (1985), Gal-Or (1986), Shapiro (1986), Ziv (1993), Raith (1996)). Similar approaches have been used to investigate the value of marketing information (Raju and Roy (2000)), information sharing in a supply chain contracting context (Lee et al. (2000), Li (2002)), and sharing security information (Gal-Or and Ghose (2005)).

In terms of the model setup, the present paper mostly resembles Sasaki (2001), in which the author uses a duopoly model where the intercept of linear demands is uncertain to show that in a Bertrand market with substitute products, one firm's information acquisition increases the other firm's incentive to acquire the same information. The present model is different in several important aspects: first, the present paper studies the equilibrium pricing variables selection, which has not been explored in the literature. Second, this stream of literature (except Sasaki (2001)) focused on multivariate normally distributed noise and signals. The current model does not impose any distributional assumptions on the pricing variables. Moreover, the information structure is completely different because this paper considers more than one pricing variable. Third, pricing variables may affect market demand in the opposite direction, which is not captured in the previous literature.

This paper adds to the theoretical pricing literature: particularly third-degree price discrimination models. This stream of literature in economics started from the seminal article of Schmalensee (1981) and Varian (1985), and has focused only on the welfare implications of price discrimination but not on the variables selection problem. In the marketing literature, researchers have investigated applications of price discrimination such as pricing based on purchase history (Villas-Boas (1999), Villas-Boas (2004), and Acquisti and Varian (2005)), spatial/targeted pricing (e.g., Thisse and Vives (1989), Shaffer and Zhang (2000), Corts (1998), Chen et al. (2001), 
Chen and Iyer (2002), Choudhary et al. (2005), Liu and Zhang (2006)), and targeted couponing (e.g., Shaffer and Zhang (1995), Shaffer and Zhang (2002)). The focus of these studies is to investigate the impacts of various pricing strategies on the profitability of firms. The present model bridges the gap between these models and business practices by explicitly modeling many pricing variables instead of one theoretical, abstract variable such as "customer type," "purchase history," "brand loyalty," "location/taste" in spatial models, and "quality preference" in vertical differentiation models.

This paper is organized as follows: Section 2 presents the setup and the results of the baseline model. Section 3 discusses the equilibrium variables selection and its applications. Concluding remarks are given in Section 4.

\section{Model}

There are two sellers A and B competing in many market segments determined by a set of pricing variables. Pricing variables are denoted by an $N$-dimensional random vector with coordinates denoted by $X_{i}$ and realizations denoted by $x_{i}$. For example, $X_{i}$ can be a binary variable, such as student status or a continuous variable such as the income of end-consumers or the financial statistics of a corporate buyer. The mean and variance of each variable are denoted by $\mu_{i}$ and $\sigma_{i}^{2}$, respectively. One neat property of this model is that no distributional assumption is imposed on the pricing variables except the correlation coefficient between any two variables is assumed to be zero. ${ }^{3}$ There are two stages of this game: (1) two sellers announce their choices of pricing variables simultaneously; (2) two sellers announce pricing in each market segment simultaneously. The solution concept is subgame perfect Nash Equilibrium.

This study models consumer demand for these two brands using the linear demand system, a model that has been used frequently in the literature. Formally, the demand of buyers with a

\footnotetext{
${ }^{3}$ If pricing variables are not orthogonal to each other, theoretically, we can apply principal component analysis to find the new coordinates for this pricing problem.
} 
demographic value vector $\left(x_{1}, \cdots x_{N}\right)$ has the following linear functional form.

$$
\begin{aligned}
D_{A}\left(x_{1}, \cdots x_{N}, p_{A}, p_{B}\right) & =\alpha_{0}+\sum_{i=1}^{N} \alpha_{i} x_{i}-\alpha_{A} p_{A}\left(x_{1}, \cdots x_{N}\right)+\alpha_{B} p_{B}\left(x_{1}, \cdots x_{N}\right) ; \\
D_{B}\left(x_{1}, \cdots x_{N}, p_{A}, p_{B}\right) & =\beta_{0}+\sum_{i=1}^{N} \beta_{i} x_{i}+\beta_{A} p_{A}\left(x_{1}, \cdots x_{N}\right)-\beta_{B} p_{B}\left(x_{1}, \cdots x_{N}\right) .
\end{aligned}
$$

where the subscripts of demand and prices denote the seller $\mathrm{A}$ and $\mathrm{B}, \alpha_{i}$ and $\beta_{i}$ are exogenous coefficients of variables, and $p_{A}(\cdot)$ and $p_{B}(\cdot)$ are product prices in each market segment. Note that $\alpha_{A}$ and $\beta_{B}$ are positive and other coefficients can be either positive or negative. Cross price coefficients, $\alpha_{B}$ and $\beta_{A}$, are both positive when two products are substitutes and both negative when two products are complements. This linear demand system encompasses models with vertical or horizontal differentiation in the literature. Applications will discussed in detail in Section 3.

For ease of exposition, I use $X_{A}$ and $X_{B}$ to denote the random vectors of variables used by seller $\mathrm{A}$ and $\mathrm{B}$, respectively. In the following analysis, the realizations of $X_{A}$ and $X_{B}$ are denoted by $x_{A}$ and $x_{B}$. Note that $X_{A}$ and $X_{B}$ can have an empty intersection set or not. For example, $X_{A}$ may include two variables (region, student status) and $X_{B}$ may include (region, gender).

Both firms are risk neutral and maximize expected profits conditional on the pricing variables. The variable costs are assumed to be zero to simplify the notations. All of the results still hold with constant variable cost. Given this setup, when firm A uses $X_{A}$, the expected revenue in each market segment is a function of $x_{A}$ and is given by

$$
R_{A}\left(x_{A}\right)=p_{A}\left(x_{A}\right) \mathbf{E}\left[D_{A}\left(x_{1}, \cdots x_{N} \mid x_{A}\right)\right]
$$

This model also considers fixed costs of price discrimination. In real-world pricing problems, there exist various kinds of costs associated with implementing price discrimination. First, sellers have to buy or collect customer information. In the next phase, sellers incur administrative costs such as menu costs, accounting costs, and pricing variables tracking costs. Price discrimination may also impose heavy (psychological) costs on the buyers arising from concerns about unfairness, aversion to complicated pricing plans, and mental costs of price discrimination by some controversial attributes. ${ }^{4}$ This paper models these costs by a constant number $F_{i}$ associated

\footnotetext{
${ }^{4} \mathrm{~A}$ model of unfairness should affect the demand of each market segment, which might be an interesting topic for future research but is beyond the scope of this paper.
} 
with each variable $X_{i}$.

Since fixed costs do not affect the second stage pricing game, sellers will choose a pricing function $p_{i}(\cdot)$ among all possible functions to maximize revenues in each market segment. The total profit can be derived by taking expectation over $X_{A}$ or $X_{B}$. Results are reported in the following sections.

\subsection{Monopoly Benchmark Case}

We first consider a monopoly benchmark case. Assuming only firm A serves the whole market, the optimal price and profit are given in the following lemma.

Lemma 1 When firm $A$ uses variable $X_{A}$ in a monopoly industry, the optimal price and revenue function is given by

$$
\begin{aligned}
p_{A}\left(x_{A}\right) & =\frac{1}{2 \alpha_{A}}\left[\mu_{A}+\sum_{i \in A} \alpha_{i}\left(x_{i}-\mu_{i}\right)\right] \\
\pi_{A} & =\frac{1}{4 \alpha_{A}}\left(\mu_{A}^{2}+\sum_{i \in A} \alpha_{i}^{2} \sigma_{i}^{2}\right)-\sum_{i \in A} F_{i}, \\
\text { where } \mu_{A} & =\sum_{i=1}^{N} \alpha_{i} \mu_{i} . \text { (expected intercept) }
\end{aligned}
$$

There are several important implications from this lemma. First, the optimal pricing function is a linear function of pricing variables, which is chosen among any possible pricing functions. In other words, the seller should use a simple pricing plan: charge customers a fixed fee $\frac{\mu_{A}}{2 \alpha_{A}}$ and a variable (usage) fee $\frac{\alpha_{i}}{2 \alpha_{A}}$. For example, eBay can charge their sellers a fixed listing fee at $\frac{\mu_{A}}{2 \alpha_{A}}$. The interpretation of $\frac{\alpha_{i}}{2 \alpha_{A}}$ depends on the pricing variable: it is a per unit fee when "the number of items sold" is the variable, or a percentage (commission) when the final auction price is the pricing variable. Note that this result is built upon the linear demand but not the distribution of pricing variables.

As to the revenue function, this model suggests a simple criterion for the monopoly seller to select pricing variables. First, the seller can consider the adoption of variables one-by-one because there is no multiplicative, interaction term in the revenue function. This nice property results from the zero correlation and the linearity of demands assumptions. Second and most 
important of all, the criterion for selecting variables is to compare $\frac{\alpha_{i}^{2} \sigma_{i}^{2}}{4 \alpha_{A}}$ with $F_{i}$. The value of price discrimination (VOPD) from using variable $X_{i}$ is $\frac{\alpha_{i}^{2} \sigma_{i}^{2}}{4 \alpha_{A}}$, which implies variables with large coefficient $\left(\alpha_{i}^{2}\right)$ or large variance $\left(\sigma_{i}^{2}\right)$ should be used for price discriminating/market segmentation. The impact of $\alpha_{A}$ on VOPD is intuitive: the larger the $\alpha_{A}$, the lower the monopoly market power is and thus the VOPD is lower.

Lastly, the improvement of revenue can be estimated by a simple formula, $\alpha_{i}^{2} \sigma_{i}^{2} / \mu_{A}^{2}$, which is similar to the squared coefficient of variation $(\sigma / \mu)$ in statistics. Firms can easily evaluate the marginal contribution of each pricing variable by calculating this number. Intuitively, $\alpha_{i}^{2} \sigma_{i}^{2}$ represents the explained variations or the source of VOPD while $\mu_{A}^{2}$ is the normalization factor as in the coefficient of variation.

\subsection{Duopoly Results}

In the duopoly case, the equilibrium prices are given in the following lemma.

Lemma 2 Suppose $\alpha_{0}$ and $\beta_{0}$ are large enough so that no market segment is closed for each firm in the equilibrium. When firms $A$ and $B$ use variables $X_{A}$ and $X_{B}$, respectively, the equilibrium prices are

$$
\begin{aligned}
p_{A}\left(x_{A}\right) & =A_{1}+\sum_{i \in A \backslash B} \frac{\alpha_{i}}{2 \alpha_{A}}\left(x_{i}-\mu_{i}\right)+\sum_{i \in A \cap B} A_{2 i}\left(x_{i}-\mu_{i}\right), \\
p_{B}\left(x_{B}\right) & =B_{1}+\sum_{i \in B \backslash A} \frac{\beta_{i}}{2 \beta_{B}}\left(x_{i}-\mu_{i}\right)+\sum_{i \in A \cap B} B_{2 i}\left(x_{i}-\mu_{i}\right), \\
\text { where } A_{1} & =\frac{2 \beta_{B} \mu_{A}+\alpha_{B} \mu_{B}}{4 \alpha_{A} \beta_{B}-\alpha_{B} \beta_{A}} \text { and } B_{1}=\frac{2 \alpha_{A} \mu_{B}+\beta_{A} \mu_{A}}{4 \alpha_{A} \beta_{B}-\alpha_{B} \beta_{A}} ; \\
A_{2 i} & =\frac{\alpha_{B} \beta_{i}+2 \beta_{B} \alpha_{i}}{4 \alpha_{A} \beta_{B}-\alpha_{B} \beta_{A}} ; \\
B_{2 i} & =\frac{2 \alpha_{A} \beta_{i}+\beta_{A} \alpha_{i}}{4 \alpha_{A} \beta_{B}-\alpha_{B} \beta_{A}}, \\
\mu_{A} & \equiv \sum_{i \in \Omega} \alpha_{i} \mu_{i} \text { and } \mu_{B} \equiv \sum_{i \in \Omega} \beta_{i} \mu_{i} .
\end{aligned}
$$

This lemma indicates that equilibrium prices are still linear functions of pricing variables but now it has three components. The first term is a constant term. The second term is a linear function of pricing variables used by firm A but not firm B (or the other way around). Note that 
this term is exactly the same as that in the monopoly case. The third term is a linear function of pricing variables used by both firms. Note that these pricing functions are mean-preserving dispersions along that dimension. For example, suppose only firm $\mathrm{A}$ uses $X_{1}$, then firm $\mathrm{B}$ is facing an expected price $\mathbf{E}\left[p_{A}\left(X_{1}\right)\right]=p_{A}\left(\mathbf{E}\left[X_{1}\right]\right)$, which is the price charged by firm A when firm A does not use $X_{1}$. This feature leads to the result that the second component is the same as that in the monopoly case. The following Theorem also shows that this feature leads to the same VOPD when only one firm uses a pricing variable in the duopoly case.

Theorem 1 When firms $A$ and $B$ use variables $X_{A}$ and $X_{B}$ respectively, the equilibrium revenues are

$$
\begin{aligned}
& \pi_{A}=\left(\alpha_{A} A_{1}^{2}+\sum_{i \in A \backslash B} \frac{\alpha_{i}^{2}}{4 \alpha_{A}} \times \sigma_{i}^{2}+\alpha_{A} \sum_{i \in A \cap B} A_{2 i}^{2} \times \sigma_{i}^{2}\right)-\sum_{i \in A} F_{i}, \\
& \pi_{B}=\left(\beta_{B} B_{1}^{2}+\sum_{i \in B \backslash A} \frac{\beta_{i}^{2}}{4 \beta_{B}} \times \sigma_{i}^{2}+\beta_{B} \sum_{i \in A \cap B} B_{2 i}^{2} \times \sigma_{i}^{2}\right)-\sum_{i \in B} F_{i} .
\end{aligned}
$$

Similar to the equilibrium price functions, the first component in the bracket, $A_{1}^{2}$ or $B_{1}^{2}$, depends on the product differentiation and firm competition but not on the selection of pricing variables. The second component is $\frac{\alpha_{i}^{2}}{4 \alpha_{A}} \times \sigma_{i}^{2}\left(\right.$ or $\left.\frac{\beta_{i}^{2}}{4 \beta_{B}} \times \sigma_{i}^{2}\right)$ which is the VOPD when only one firm uses variable $X_{i}$. This value is exactly the same as that in the monopoly case. Intuitively, when only one firm uses a variable, the other firm's pricing along that dimension will be the same as shown in Lemma 2. Since the opponent will not react to the pricing policy change, the equilibrium VOPD is the same as that in the monopoly case. The last component represents the VOPD when both firms use $X_{i}$. The expression of this coefficient depends on several parameters and is more complicated. The next section will discuss special cases and shed more lights on this term.

Theorem 1 also suggests that the revenue and cost from pricing based on each variable do not have interaction terms (i.e., there is no multiplicative term of any two variables). In other words, each firm's optimal response can be greatly simplified. In this game, each firm has to consider $2^{N}$ possible variables selections and the payoff matrix is a $2^{N}$-by-2 $2^{N}$ matrix. With the independence property, this game can be decomposed into $N$ 2-by-2 payoff matrix. A typical payoff matrix is illustrated in Table 1 . In the following analysis, I shall use the notation $\left(\varnothing, X_{i}\right)$ 


\begin{tabular}{|c|c|c|}
\hline (Additional Profit) & Firm B uses $X_{i}$ & Firm B does not use $X_{i}$ \\
\hline Firm A uses $X_{i}$ & $\alpha_{A} A_{2 i}^{2} \sigma_{i}^{2}-F_{i}, \beta_{B} B_{2 i}^{2} \sigma_{i}^{2}-F_{i}$ & $\frac{\alpha_{i}^{2}}{4 \alpha_{A}} \sigma_{i}^{2}-F_{i}, 0$ \\
\hline Firm A does not use $X_{i}$ & $0, \frac{\beta_{i}^{2}}{4 \beta_{B}} \sigma_{i}^{2}-F_{i}$ & 0,0 \\
\hline
\end{tabular}

Table 1: Equilibrium Additional Profits from Pricing Based on $X_{i}$

to denote the equilibrium in which the first element $\varnothing$ means firm A does not use $X_{i}$ and the second element $X_{i}$ means firm B uses $X_{i}$.

Corollary 1 This $2^{N}$-by-2 $2^{N}$ variables selection game is equivalent to $N$ 2-by-2 single variable selection game.

\section{Equilibrium Variables Selection}

In the general case presented in the previous section, all variable selections are possible in the equilibrium. To shed more lights on the new research questions, it is necessary to put some restrictions on the parameters. This section considers two cases: (1) the symmetric case and (2) the firm-specific variable case (pricing variables affect only one firm's demand). Following each case, I will provide a stylized example to highlight key findings.

\subsection{Symmetric Case}

This paper defines symmetric firms by assuming $\alpha_{i}= \pm \beta_{i}, \alpha_{A}=\beta_{B}$, and $\alpha_{B}=\beta_{A}$. It is worth mention that when $\alpha_{i}=\beta_{i}$, a pricing variable affects both firms' demand in the same direction with the same magnitude. In other words, this type of variables identifies what is the market segment that has higher demand for both firms' products. In contrast, when $\alpha_{i}=-\beta_{i}$, a pricing variable affects the duopoly demand in the opposite direction with the same magnitude. For example, in the case of Netflix and Blockbuster, some customers used to rent a DVD online whereas the others used to rent a DVD offline depending on where they lived or the convenience of their Internet access.

These symmetric assumptions greatly reduce the complexity of the equilibrium results. By Theorem 1 and Table 1, there are only three regions of NEs depending on the value of fixed cost, 
$F_{i}$. When the fixed cost is very high (or very low), it is a dominant strategy for both firms not to use (or to use) variable $X_{i}$. There are only two subcases in the intermediate region and the result is summarized in the following proposition.

Proposition 1 (1) When $F_{i} / \sigma_{i}^{2} \geq \max \left[\alpha_{A} A_{2 i}^{2}, \frac{\alpha_{i}^{2}}{4 \alpha_{A}}\right]$, the unique $N E$ is ( $\left.\varnothing, \varnothing\right)$.

(2) When $\min \left[\alpha_{A} A_{2 i}^{2}, \frac{\alpha_{i}^{2}}{4 \alpha_{A}}\right] \leq F_{i} / \sigma_{i}^{2} \leq \max \left[\alpha_{A} A_{2 i}^{2}, \frac{\alpha_{i}^{2}}{4 \alpha_{A}}\right]$,

(2-1) There are two NEs: either $(\varnothing, \varnothing)$ or $\left(X_{i}, X_{i}\right)$ is an equilibrium when two products are substitutes $\left(\alpha_{B}=\beta_{A}>0\right)$, and $X_{i}$ affects the demand functions in the same direction $\left(\alpha_{i}=\beta_{i}\right)$, or when two products are complement goods $\left(\alpha_{B}=\beta_{A}<0\right)$, and $X_{i}$ affects the demand functions in the opposite direction.

(2-2) There are two NEs: either $\left(\varnothing, X_{i}\right)$ or $\left(X_{i}, \varnothing\right)$ is an equilibrium when two products are substitutes $\left(\alpha_{B}=\beta_{A}>0\right)$, and $X_{i}$ affects the demand functions in the opposite direction $\left(\alpha_{i}=-\beta_{i}\right)$, or when two products are complement goods $\left(\alpha_{B}=\beta_{A}<0\right)$, and $X_{i}$ affects the demand functions in the same direction.

(3) When $F_{i} / \sigma_{i}^{2} \leq \min \left[\alpha_{A} A_{2 i}^{2}, \frac{\alpha_{i}^{2}}{4 \alpha_{A}}\right]$, the unique $N E$ is $\left(X_{i}, X_{i}\right)$.

Cases (1) and (3) are intuitive since $F_{i} / \sigma_{i}^{2}$ measures the cost/benefit in this model. The intuition of (2-1) is as follows: when only firm A price discriminates on $X_{i}$, firm A will raise its price in the strong market. If firm B does not use $X_{i}$, it will keep its price at the original level, which will drag down firm A's price because of product competition. When firm B also uses $X_{i}$, firm B will also raise its price in firm A's strong market, which will further elevate firm A's equilibrium price in the strong market because these two products are substitutes. As a result, the equilibrium prices will be more dispersed when both firms use $X_{i}$ compared with the case in which only firm A uses $X_{i}$. Since the revenue is a quadratic function of equilibrium prices, the more dispersed the price is, the higher the equilibrium revenue is. ${ }^{5}$ In other words, this type of variable can coordinate the pricing strategy of a duopoly. A duopoly will raise prices in a profitable market and lower prices in a non-profitable market. The gain in the profitable market

\footnotetext{
${ }^{5}$ Recall that the equilibrium price is a mean-preserving function of the equilibrium uniform price. In other words, the equilibrium prices always have the same mean no matter which the metrics are chosen by firms A or B. Also, the equilibrium revenue is equal to the squared prices. By Jensen's inequality, the more dispersed the equilibrium prices are, the higher the equilibrium revenue will be.
} 
will dominate the loss in the weak market, leading to higher equilibrium revenue. For example, suppose two firms identify a market segment that is very lucrative. If only firm A raises prices in that market, firm A cannot improve profits significantly because of firm B's low price. If firm B also raises price in that segment, then both firms can benefit from relaxing competition in that segment and earn more profits in the equilibrium.

In contrast, when the variable affects demand functions in the opposite direction as in (22), firm B will lower its price in firm A's strong market when both firms use $X_{i}$. As a result, the equilibrium prices will be less dispersed when both firms use $X_{i}$ compared with the case in which only firm A use $X_{i}$. This intuition is similar to the idea explored in marketing literature that targeted pricing or targeted coupons may lead to lower equilibrium profits. The case of complements is also interesting. The equilibrium effects are reversed because the best response of firms has different directional effects when the other firm raises or lowers prices. For example, when both firms use $X_{i}$, firm B still raises prices in firm A's strong market segments. However, the higher prices of firm B put pressure on firm A's pricing because two products are complements (e.g., if the inkjet is already expensive, printer cannot be too expensive). As a result, the equilibrium price will be lower rather than higher as in the substitutes case.

\subsection{Application: Horizontal Differentiation}

There are two sellers $A$ and $B$ selling horizontally differentiated products. For example, firm A can be Blockbuster and firm B can be Netflix.com. There are two pricing variables, $X_{1}$ and $X_{2}$. Customers are grouped into several market segments based on these two demographic variables. $X_{1}$ indicates how far a customer lives from a Blockbuster's store. $X_{2}$ indicates wether or not a customer likes to shop online. In each market, there are two sellers $A$ and $B$ located at the two ends of a straight line $[-1,1]$. The utility function of a buyer located at $x$ with demographic values $\left(x_{1}, x_{2}\right)$ to buy from each seller is assumed to be

$$
\begin{aligned}
& U_{A}=K-\frac{1}{2}[x-(-1)]-p_{A} ; \\
& U_{B}=K-\frac{1}{2}(1-x)-p_{B},
\end{aligned}
$$




\begin{tabular}{|c|c|c|}
\hline (Additional Profit) & Firm B uses $X_{i}$ & Firm B does not use $X_{i}$ \\
\hline Firm A uses $X_{i}$ & $\frac{1}{9} \sigma_{i}^{2}-F_{i}, \frac{1}{9} \sigma_{i}^{2}-F_{i}$ & $\sigma_{i}^{2}-F_{i}, 0$ \\
\hline Firm A does not use $X_{i}$ & $0, \sigma_{i}^{2}-F_{i}$ & 0,0 \\
\hline
\end{tabular}

Table 2: Equilibrium Additional Profits from Pricing Based on $X_{i}$

where $K$ is a constant large enough so that every customer buys from either firm $A$ or $B, x$ is the location of that customer and is uniformly distributed in $\left[X_{1}+X_{2}-\frac{1}{2}, X_{1}+X_{2}+\frac{1}{2}\right] .{ }^{6}$ It can be verified that traveling cost does affect the results and thus is assumed to be $1 / 2$ for simplicity. Given this setup, it follows that the demand of each firm in each market segment is

$$
\begin{aligned}
& D_{A}=-X_{1}-X_{2}+\frac{1}{2}-p_{A}+p_{B} \\
& D_{B}=X_{1}+X_{2}+\frac{1}{2}+p_{A}-p_{B} .
\end{aligned}
$$

Compared with the baseline model, it follows that $\alpha_{1}=-1, \beta_{1}=-1, \alpha_{2}=1, \beta_{2}=1$, and $\alpha_{A}=\alpha_{B}=\beta_{A}=\beta_{B}=1$. By Theorem (1), the payoff matrices of using $X_{1}$ and $X_{2}$ are given in Table 2 .

When only one firm uses either $X_{1}$ or $X_{2}$, the value of price discrimination is $\frac{1}{4} \sigma_{1}^{2}$. When both firms uses $X_{1}$, the value of price discrimination is $\frac{1}{9} \sigma_{1}^{2}$. This comes from the targeted pricing effect discussed in Section 3. As a result, with moderate fixed costs of price discrimination, only one firm should use a specific type of discounting (price discrimination) in the equilibrium. Note that there are multiple equilibria of the full game: (1) firm A uses $X_{1}$ and $X_{2} ;(2)$ firm B uses $X_{1}$ and $X_{2}$; (1) firm A uses $X_{1}$ and firm B uses $X_{2}$; (2) firm B uses $X_{1}$ and firm A uses $X_{2}$. This partly explains why it is easy to find inconsistent pricing variables for similar products in practice. In the real world, if there are additional fixed costs associated with the number of pricing variables, then only cases (3) and (4) are feasible, in which each firm uses different types of discounting strategy to avoid price competition.

${ }^{6} X_{1}-X_{2}-\frac{1}{2} \geq-1$ and $X_{1}+X_{2}+\frac{1}{2} \leq 1$ are assumed to hold in all market segments in this example. All other assumptions in Section 2 still apply here. 


\subsection{Firm Specific Variable Case}

This section considers the case in which only one firm's demand is affected by a variable. Formally, let $\alpha_{i}>0$ and $\beta_{i}=0$. Again, by Theorem 1 and Table 1, the equilibrium results can be derived and are summarized as follows

Proposition 2 (1) When $4 \alpha_{A} \beta_{B}-\beta_{A}^{2} \geq 0$ and $2\left|\beta_{A}\right| \sqrt{\alpha_{A} \beta_{B}} \leq 4 \alpha_{A} \beta_{B}-\alpha_{B} \beta_{A}$, the NEs in each region are

(1-1) When $F_{i} / \sigma_{i}^{2} \geq \frac{1}{4 \alpha_{A}} \alpha_{i}^{2}$, the unique $N E$ is $(\varnothing, \varnothing)$.

(1-2) When $\frac{1}{4 \alpha_{A}} \alpha_{i}^{2} \geq F_{i} / \sigma_{i}^{2} \geq \beta_{B} B_{2 i}^{2}$, the unique $N E$ is $\left(X_{i}, \oslash\right)$.

(1-3) When $F_{i} / \sigma_{i}^{2} \leq \beta_{B} B_{2 i}^{2}$, the unique $N E$ is $\left(X_{i}, X_{i}\right)$.

(2) Otherwise, the NEs in each region are

(1-1) When $F_{i} / \sigma_{i}^{2} \geq \min \left[\beta_{B} B_{2 i}^{2}, \alpha_{A} A_{2 i}^{2}\right]$, the unique $N E$ is $(\varnothing, \varnothing)$.

(1-2) When $\min \left[\beta_{B} B_{2 i}^{2}, \alpha_{A} A_{2 i}^{2}\right] \geq F_{i} / \sigma_{i}^{2} \geq \frac{1}{4 \alpha_{A}} \alpha_{i}^{2}$, there are two NEs: $\left(X_{i}, X_{i}\right)$ or $(\varnothing, \varnothing)$.

(1-3) When $F_{i} / \sigma_{i}^{2} \leq \frac{1}{4 \alpha_{A}} \alpha_{i}^{2}$, the unique $N E$ is $\left(X_{i}, X_{i}\right)$.

Intuitively, it is highly possible that only firm A will use the variable in the equilibrium $\left(X_{i}\right.$, $\varnothing)$ since the variable affects only its demand. However, there are many cases in which firm B will also use variable $X_{i}$ when firm A uses $X_{i}$. The intuition is that although $X_{i}$ does not affect firm B's demand directly, firm A's price will affect firm B's demand and hence its best response. When firm A charges different prices in different markets, firm B may also benefit from changing its prices in each market accordingly, which is called information spillover effect in this paper. When the fixed cost is small enough, the benefit from information spillover effect dominates and hence firm B will also use that variable in the equilibrium.

\subsection{Application: Vertical Differentiation}

There are two sellers $A$ and $B$ selling products with different quality levels, $q_{A}$ and $q_{B}$, respectively $\left(q_{A}>q_{B}\right)$. For example, Firm A is Orbitz.com or Expedia.com who offers travel items at regular prices whereas Firms B is Priceline.com or Hotwire.com who offers non-flexible air, hotel, or rental cars at discounted prices. Consider a simple case with two variables, $X_{1}$ and $X_{2}$. $X_{1}$ is the 
frequency with which a customer traveled during the last year. $X_{2}$ is the frequency with which a customer used the discounted services provided by Firm B. The utility function of a buyer with demographic values $\left(x_{1}, x_{2}\right)$ to buy from each seller is assumed to be

$$
\begin{aligned}
& U_{A}=x_{1}+\theta q_{A}-p_{A} ; \\
& U_{B}=x_{1}+\theta q_{B}-p_{B},
\end{aligned}
$$

where $\theta$ models the heterogeneity of customers and is the marginal willingness-to-pay of unit quality. Consistent with the literature, $\theta$ is assumed to be uniformly distributed in $\left[x_{2}-\frac{1}{2}\right.$, $\left.x_{2}+\frac{1}{2}\right]$. In other words, $x_{1}$ models the fixed valuation of travel items provided by both firms whereas $x_{2}$ models the expected quality preference of customers in each market segment.

Assuming market is always not fully covered in all realizations of $\left(x_{1}, x_{2}\right)$, the demand of each seller is given by

$$
\begin{aligned}
& D_{A}=x_{2}+\frac{1}{2}-\frac{p_{A}-p_{B}}{q_{A}-q_{B}} ; \\
& D_{B}=\frac{p_{A}-p_{B}}{q_{A}-q_{B}}-\frac{p_{B}-x_{1}}{q_{B}} .
\end{aligned}
$$

Compared with the baseline model, it follows that $\alpha_{1}=0, \beta_{1}=1 / q_{B}, \alpha_{2}=1, \beta_{2}=0$, and $\alpha_{A}=\alpha_{B}=\beta_{A}=1 /\left(q_{A}-q_{B}\right)=q_{B} \beta_{B} / q_{A}$. For ease of comparison, assume $F_{1} / \sigma_{1}^{2}=F_{2} / \sigma_{2}^{2}$. Applying Proposition 2 leads to

Proposition 3 In this model, $4 \alpha_{A} \beta_{B}-\beta_{A}^{2} \geq 0$ and $2\left|\beta_{A}\right| \sqrt{\alpha_{A} \beta_{B}} \leq 4 \alpha_{A} \beta_{B}-\alpha_{B} \beta_{A}$ both holds.

The NEs regarding $X_{1}$ in each region are

(1-1) When $F_{i} / \sigma_{i}^{2} \geq \frac{\left(q_{A}-q_{B}\right)}{4 q_{A} q_{B}}$, the unique $N E$ is $(\varnothing, \varnothing)$.

(1-2) When $\frac{\left(q_{A}-q_{B}\right)}{4 q_{A} q_{B}} \geq F_{i} / \sigma_{i}^{2} \geq \frac{\left(q_{A}-q_{B}\right)}{\left(4 q_{A}-q_{B}\right)^{2}}$, the unique $N E$ is $\left(\oslash, X_{1}\right)$.

(1-3) When $F_{i} / \sigma_{i}^{2} \leq \frac{\left(q_{A}-q_{B}\right)}{\left(4 q_{A}-q_{B}\right)^{2}}$, the unique $N E$ is $\left(X_{1}, X_{1}\right)$.

The NEs regarding $X_{2}$ in each region are

(1-1) When $F_{i} / \sigma_{i}^{2} \geq \frac{\left(q_{A}-q_{B}\right)}{4}$, the unique NE is $(\varnothing, \varnothing)$.

(1-2) When $\frac{\left(q_{A}-q_{B}\right)}{4} \geq F_{i} / \sigma_{i}^{2} \geq \frac{\left(q_{A}-q_{B}\right) q_{B} q_{A}}{\left(4 q_{A}-q_{B}\right)^{2}}$, the unique NE is $\left(X_{2}, \oslash\right)$.

(1-3) When $F_{i} / \sigma_{i}^{2} \leq \frac{\left(q_{A}-q_{B}\right) q_{B} q_{A}}{\left(4 q_{A}-q_{B}\right)^{2}}$, the unique $N E$ is $\left(X_{2}, X_{2}\right)$.

First, in the intermediate cases, the high quality firm $\mathrm{A}$ will use $X_{2}$ and the low quality firm B will use $X_{1}$ in this model. This model suggests that when the pricing variable affects only the 
overall WTP, the lower quality firm has greater incentives to use it. When a pricing variable affects the distribution of quality preferences, the higher quality firm has greater incentives to

use it. Second, when only firm A adopts $X_{2}$, its VOPD is $\frac{\left(q_{A}-q_{B}\right)}{4} \sigma_{2}^{2}$. This term is increasing in $q_{A}$ and decreasing in $q_{B}$. When only firm B adopts $X_{1}$, its VOPD is $\frac{\left(q_{A}-q_{B}\right)}{4 q_{A} q_{B}} \sigma_{2}^{2}$. This term is also increasing in $q_{A}$ and decreasing in $q_{B}$. The intuition is that the value of price discrimination in this case is determined by the size of the vertical differentiation $\left(q_{A}-q_{B}\right)$. The larger the difference is, the more differentiation there is between two firms, and the value of price discrimination is higher.

\section{Concluding Remarks}

This paper examines the properties of a profit-maximizing set of variables for third-degree price discrimination. In the monopoly case, the seller should use pricing variables with high explanatory power of demand: variables with large demand coefficients or high variance. In the duopoly case, that criterion still applies but the value of price discrimination will be affected by competition, too. This model shows that when only one firm uses a pricing variable, the value of price discrimination is the same as in the monopoly case. When both firms use a pricing variable, the value of price discrimination is higher when two products are substitutes and that variable affects demands in the same direction. In contrast, the value of price discrimination is lower when two firms sell substitutes and that variable affects demands in the opposite direction.

Several related issues may require further analysis. First and foremost, the robustness of the results under different demand curves assumptions. There are two directions to generalize the demand functions: (1) letting pricing variables affect demand coefficients; (2) nonlinear demand curve. Both routes are important directions for future investigation but are beyond the scope of this paper. Second, this study considers the case of third-degree price discrimination and excludes customer self-selection or arbitrage between market segments. A model that incorporates these features is a multi-dimensional nonlinear pricing model, which is notoriously analytically complicated. Lastly, this study assumes that no firm is competed out of any market segment. Relaxing this assumption may lead to messy algebraic results and obfuscate the key insights provided by the current model. But, the gain would be an analysis of the entry and exit strategies 
of firms in each market segment.

\section{Appendix: Proofs}

\subsection{Proof of Lemma 1}

Suppose the monopoly chooses to use $x_{1}, \cdots, x_{m}$ for market segmentation and denote this vector of variables by $x_{A}$. The demand curve of this monopoly is given by

$$
D_{A}\left(x_{A}, p_{A}\right)=\alpha_{0}+\sum_{i=1}^{m} \alpha_{i} x_{i}+\sum_{i=m+1}^{N} \alpha_{i} \mu_{i}-\alpha_{A} p_{A}\left(x_{A}\right)
$$

The objective function is simply

$$
\pi_{A}\left(x_{A}\right)=D_{A}\left(x_{A}, p_{A}\right) \times p_{A}\left(x_{A}\right)
$$

The first order condition yields

$$
\alpha_{0}+\sum_{i=1}^{m} \alpha_{i} x_{i}+\sum_{i=m+1}^{N} \alpha_{i} \mu_{i}-2 \alpha_{A} p_{A}\left(x_{A}\right)=0 .
$$

The optimal price in the main text is derived after rearranging terms. The first order condition also implies

$$
D_{A}\left(x_{A}, p_{A}\right)=\alpha_{A} p_{A}\left(x_{A}\right)
$$

As a result, it follows that

$$
\pi_{A}\left(x_{A}\right)=\alpha_{A} p_{A}^{2}\left(x_{A}\right)=\frac{1}{4 \alpha_{A}}\left[\mu_{A}+\sum_{i \in A} \alpha_{i}\left(x_{i}-\mu_{i}\right)\right]^{2} .
$$

Taking expectation on this term yields the result of this lemma.

\subsection{Proof of Lemma 2}

The first order condition leads to

$$
\mathbf{E}_{X_{i \in A^{C}}}\left[D_{A}\left(x_{1}, \cdots x_{N}, p_{A}, p_{B}\right)\right]-\alpha_{A} p_{A}\left(x_{A}\right)=0 .
$$


The first term is simply the expected demand over the variables that are not used by firm A. I use the subscript for expectations taking over $X_{i \in A^{C}}$. The set $A^{C}$ denotes the complement of set $A$ and thus is the set of variables not used by firm A. The expected demand and the FOC can be rewritten as

$$
\alpha_{0}+\sum_{i \in A} \alpha_{i} x_{i}+\sum_{i \in A^{C}} \alpha_{i} \mu_{i}-\alpha_{A} p_{A}\left(x_{A}\right)+\alpha_{B} \mathbf{E}_{X_{i \in A^{C}}}\left[p_{B}\left(x_{B}\right)\right]-\alpha_{A} p_{A}\left(x_{A}\right)=0
$$

Similarly, the first order condition from firm B's profit optimization problem is given by

$$
\beta_{0}+\sum_{i \in B} \beta_{i} x_{i}+\sum_{i \in B^{C}} \beta_{i} \mu_{i}+\beta_{A} \mathbf{E}_{X_{i \in B^{C}}}\left[p_{A}\left(x_{A}\right)\right]-2 \beta_{B} p_{B}\left(x_{B}\right)=0 .
$$

Note that the expectations are effectively only on set $B \backslash A$ and $A \backslash B$ in two equations, respectively. $B \backslash A$ means the set of variables used by firm $\mathrm{B}$ but not by firm $\mathrm{A}$. In other words, both expected price functions are functions of $x_{A \cap B}$. The next step is to derive expected pricing functions of $x_{A \cap B}$. Taking expectations on both sides with respect to $X_{A \backslash B}$ and $X_{B \backslash A}$ and note that this step does not affect the expected price functions, it follows that

$$
\alpha_{0}+\sum_{i \in A \cap B} \alpha_{i} x_{i}+\sum_{i \in(A \cap B)^{C}} \alpha_{i} \mu_{i}+\alpha_{B} \mathbf{E}_{X_{i \in A^{C}}}\left[p_{B}\left(x_{B}\right)\right]=2 \alpha_{A} \mathbf{E}_{X_{i \in B} C}\left[p_{A}\left(x_{A}\right)\right]
$$

and

$$
\beta_{0}+\sum_{i \in A \cap B} \beta_{i} x_{i}+\sum_{i \in(A \cap B)^{C}} \beta_{i} \mu_{i}+\beta_{A} \mathbf{E}_{X_{i \in B^{C}}}\left[p_{A}\left(x_{A}\right)\right]=2 \beta_{B} \mathbf{E}_{X_{i \in A^{C}}}\left[p_{B}\left(x_{B}\right)\right] .
$$

Equivalently, we can use $\mu_{A}$ and $\mu_{B}$ to simplify the LHS of these equations and it follows that

$$
\mu_{A}+\sum_{i \in A \cap B} \alpha_{i}\left(x_{i}-\mu_{i}\right)+\alpha_{B} \mathbf{E}_{X_{i \in A^{C}}}\left[p_{B}\left(x_{B}\right)\right]=2 \alpha_{A} \mathbf{E}_{X_{i \in B^{C}}}\left[p_{A}\left(x_{A}\right)\right]
$$

and

$$
\mu_{B}+\sum_{i \in A \cap B} \beta_{i}\left(x_{i}-\mu_{i}\right)+\beta_{A} \mathbf{E}_{X_{i \in B^{C}}}\left[p_{A}\left(x_{A}\right)\right]=2 \beta_{B} \mathbf{E}_{X_{i \in A^{C}}}\left[p_{B}\left(x_{B}\right)\right]
$$

Solving these two equations yields

$$
\begin{aligned}
& \mathbf{E}_{X_{i \in B} C}\left[p_{A}\left(x_{A}\right)\right]=\frac{\alpha_{B} \mu_{B}+2 \beta_{B} \mu_{A}+\sum_{i \in A \cap B}\left(\alpha_{B} \beta_{i}+2 \beta_{B} \alpha_{i}\right)\left(x_{i}-\mu_{i}\right)}{4 \alpha_{A} \beta_{B}-\alpha_{B} \beta_{A}} \\
& \mathbf{E}_{X_{i \in A} C}\left[p_{B}\left(x_{B}\right)\right]=\frac{2 \alpha_{A} \mu_{B}+\beta_{A} \mu_{A}+\sum_{i \in A \cap B}\left(2 \alpha_{A} \beta_{i}+\beta_{A} \alpha_{i}\right)\left(x_{i}-\mu_{i}\right)}{4 \alpha_{A} \beta_{B}-\alpha_{B} \beta_{A}}
\end{aligned}
$$

After substituting these two expected prices in (6) and (7) and rearranging terms, we have 


$$
\begin{aligned}
& p_{A}\left(x_{A}\right)=\frac{1}{2 \alpha_{A}}\left[\mu_{A}+\sum_{i \in A} \alpha_{i}\left(x_{i}-\mu_{i}\right)+\alpha_{B} \cdot \frac{2 \alpha_{A} \mu_{B}+\beta_{A} \mu_{A}+\sum_{i \in A \cap B}\left(2 \alpha_{A} \beta_{i}+\beta_{A} \alpha_{i}\right)\left(x_{i}-\mu_{i}\right)}{4 \alpha_{A} \beta_{B}-\alpha_{B} \beta_{A}}\right], \\
& p_{B}\left(x_{B}\right)=\frac{1}{2 \beta_{B}}\left[\mu_{B}+\sum_{i \in B} \beta_{i}\left(x_{i}-\mu_{i}\right)+\beta_{A} \cdot \frac{\alpha_{B} \mu_{B}+2 \beta_{B} \mu_{A}+\sum_{i \in A \cap B}\left(\alpha_{B} \beta_{i}+2 \beta_{B} \alpha_{i}\right)\left(x_{i}-\mu_{i}\right)}{4 \alpha_{A} \beta_{B}-\alpha_{B} \beta_{A}}\right] .
\end{aligned}
$$

This lemma establishes after rearranging terms.

\subsection{Proof of Theorem 1}

The first order conditions lead to

$$
\mathbf{E}_{X_{i \in A} C}\left[D_{A}\left(x_{1}, \cdots x_{N}, p_{A}, p_{B}\right)\right]-p_{A}\left(x_{A}\right) \cdot \frac{\mathbf{E}_{X_{i \in A^{C}}}\left[D_{A}\left(x_{1}, \cdots x_{N} \mid x_{A}\right)\right]}{\partial p_{A}\left(x_{A}\right)}=0
$$

which is equivalent to

$$
\mathbf{E}_{X_{i \in A^{C}}}\left[D_{A}\left(x_{1}, \cdots x_{N}, p_{A}, p_{B}\right)\right]=\alpha_{A} p_{A}\left(x_{A}\right)
$$

Similarly,

$$
\mathbf{E}_{X_{i \in B^{C}}}\left[D_{B}\left(x_{1}, \cdots x_{N}, p_{A}, p_{B}\right)\right]=\beta_{B} p_{B}\left(x_{B}\right)
$$

These equations show the equation between expected demand and product prices. Substituting for the expected demands in the objective functions, it follows that

$$
\begin{aligned}
& R_{A}\left(x_{A}\right)=\alpha_{A} \cdot p_{A}\left(x_{A}\right)^{2} \\
& R_{B}\left(x_{B}\right)=\beta_{B} \cdot p_{B}\left(x_{B}\right)^{2} .
\end{aligned}
$$

Note that only the squared terms (e.g., $\left.\mathrm{E}\left[\left(x_{i}-\mu_{i}\right)^{2}\right]\right)$ of $p_{A}\left(x_{A}\right)^{2}$ will be nonzero because $\mathrm{E}\left[\left(x_{i}-\mu_{i}\right)\right]=0$ and $\mathrm{E}\left[\left(x_{i}-\mu_{i}\right)\left(x_{j}-\mu_{j}\right)\right]=0, \forall i \neq j$. The results of equilibrium revenue can be established after taking expectation on $R_{A}\left(x_{A}\right)$ and $R_{B}\left(x_{B}\right)$, respectively.

\subsection{Proof of Proposition 1}

By Theorem $1, \alpha_{i}= \pm \beta_{i}, \alpha_{A}=\beta_{B}$, and $\alpha_{B}=\beta_{A}$.Hence,

$$
\alpha_{A} A_{2 i}^{2}=\alpha_{A}\left(\frac{ \pm \alpha_{B}+2 \alpha_{A}}{4 \alpha_{A}^{2}-\alpha_{B}^{2}}\right)^{2} \alpha_{i}^{2} \text { and } \beta_{B} B_{2 i}^{2}=\beta_{B}\left(\frac{ \pm 2 \alpha_{A}+\alpha_{B}}{4 \alpha_{A}^{2}-\alpha_{B}^{2}}\right)^{2} \alpha_{i}^{2} .
$$


By Table 1, it is straightforward to verify that (1) when $\alpha_{A} A_{2 i}^{2}>\frac{\alpha_{i}^{2}}{4 \alpha_{A}}$, then there are two NEs: either $(\varnothing, \varnothing)$ or $\left(X_{i}, X_{i}\right)$ is an equilibrium; $(2)$ otherwise, there are two other NEs: either $\left(\varnothing, X_{i}\right)$ or $\left(X_{i}, \varnothing\right)$ is an equilibrium. The proof of this claim is as follows:

$\alpha_{A} A_{2 i}^{2}>\frac{\alpha_{i}^{2}}{4 \alpha_{A}}$ if and only if

$$
\alpha_{A}\left(\frac{ \pm \alpha_{B}+2 \alpha_{A}}{4 \alpha_{A}^{2}-\alpha_{B}^{2}}\right)^{2} \alpha_{i}^{2}-\frac{\alpha_{i}^{2}}{4 \alpha_{A}}>0
$$

which can be simplified to

$$
\left\{\begin{array}{ccc}
\frac{\left(4 \alpha_{A}-\alpha_{B}\right) \alpha_{B} \alpha_{i}^{2}}{4\left(2 \alpha_{A}-\alpha_{B}\right)^{2} \alpha_{A}}>0, & \text { if } \quad \alpha_{i}=\beta_{i} \\
-\frac{\left(4 \alpha_{A}+\alpha_{B}\right) \alpha_{B} \alpha_{i}^{2}}{4\left(\alpha_{B}+2 \alpha_{A}\right) \alpha_{A}}>0, & \text { if } \quad \alpha_{i}=-\beta_{i}
\end{array}\right.
$$

Since $\alpha_{A}>0$ and $\alpha_{A} \geq \alpha_{B}$, it follows that

$$
\left\{\begin{array}{c}
\alpha_{B}>0, \text { if } \quad \alpha_{i}=\beta_{i} \\
\alpha_{B}<0, \text { if } \quad \alpha_{i}=-\beta_{i}
\end{array}\right.
$$

This establishes the proposition.

\subsection{Proof of Proposition 2}

To characterize the equilibrium variables selection, we need to find the orderings of the following four revenues: $\alpha_{A} A_{2 i}^{2} \sigma_{i}^{2}, \frac{\alpha_{i}^{2}}{4 \alpha_{A}} \sigma_{i}^{2}, \beta_{B} B_{2 i}^{2} \sigma_{i}^{2}$, and $\frac{\beta_{i}^{2}}{4 \beta_{B}} \sigma_{i}^{2}$. Note that by assumption, $\beta_{i}=0$. Since all other numbers are positive in this case, we only need to compare the other three variables.

(1) $\alpha_{A} A_{2 i}^{2} \sigma_{i}^{2} \geq \beta_{B} B_{2 i}^{2} \sigma_{i}^{2}$ if and only if

$$
\begin{aligned}
& \alpha_{A} A_{2 i}^{2}-\beta_{B} B_{2 i}^{2} \geq 0 \\
\Leftrightarrow & \frac{\left(4 \alpha_{A} \beta_{B}-\beta_{A}^{2}\right) \beta_{B} \alpha_{i}^{2}}{\left(4 \alpha_{A} \beta_{B}-\alpha_{B} \beta_{A}\right)^{2}} \geq 0 \\
\Leftrightarrow & \left(4 \alpha_{A} \beta_{B}-\beta_{A}^{2}\right) \geq 0 .
\end{aligned}
$$

(2) $\alpha_{A} A_{2 i}^{2} \sigma_{i}^{2} \geq \frac{\alpha_{i}^{2}}{4 \alpha_{A}} \sigma_{i}^{2}$ if and only if

$$
\begin{aligned}
& \alpha_{A} A_{2 i}^{2}-\frac{\alpha_{i}^{2}}{4 \alpha_{A}} \geq 0 \\
\Leftrightarrow & \frac{\left(8 \alpha_{A} \beta_{B}-\alpha_{B} \beta_{A}\right) \alpha_{B} \beta_{A} \alpha_{i}^{2}}{4\left(4 \alpha_{A} \beta_{B}-\alpha_{B} \beta_{A}\right)^{2} \alpha_{A}} \geq 0 \\
\Leftrightarrow & \alpha_{B} \beta_{A} \geq 0,
\end{aligned}
$$


where the last inequality results from $8 \alpha_{A} \beta_{B}-\alpha_{B} \beta_{A}>\alpha_{A} \beta_{B}-\alpha_{B} \beta_{A} \geq 0 . \alpha_{B} \beta_{A} \geq 0$ is always true by assumption.

(3) $\beta_{B} B_{2 i}^{2} \sigma_{i}^{2} \geq \frac{\alpha_{i}^{2}}{4 \alpha_{A}} \sigma_{i}^{2}$ if and only if

$$
\begin{aligned}
& \beta_{B} B_{2 i}^{2}-\frac{\alpha_{i}^{2}}{4 \alpha_{A}} \geq 0 \\
\Leftrightarrow & -\frac{\left(16 \alpha_{A}^{2} \beta_{B}^{2}-4 \alpha_{A} \beta_{A}^{2} \beta_{B}-8 \alpha_{A} \alpha_{B} \beta_{A} \beta_{B}+\alpha_{B}^{2} \beta_{A}^{2}\right) \alpha_{i}^{2}}{4\left(4 \alpha_{A} \beta_{B}-\alpha_{B} \beta_{A}\right)^{2} \alpha_{A}} \geq 0 \\
\Leftrightarrow & -\left(16 \alpha_{A}^{2} \beta_{B}^{2}-8 \alpha_{A} \alpha_{B} \beta_{A} \beta_{B}+\alpha_{B}^{2} \beta_{A}^{2}-4 \alpha_{A} \beta_{A}^{2} \beta_{B}\right) \geq 0 . \\
\Leftrightarrow & 4 \alpha_{A} \beta_{A}^{2} \beta_{B} \geq\left(4 \alpha_{A} \beta_{B}-\alpha_{B} \beta_{A}\right)^{2} . \\
\Leftrightarrow & 2\left|\beta_{A}\right| \sqrt{\alpha_{A} \beta_{B}} \geq 4 \alpha_{A} \beta_{B}-\alpha_{B} \beta_{A} .
\end{aligned}
$$

As a result, 8 and 9 determine the orderings of the revenues. Once the ordering is determined, the equilibrium can be derived by Table 1 . The three cases are: (1) When $4 \alpha_{A} \beta_{B}-\beta_{A}^{2} \geq 0$ and $2\left|\beta_{A}\right| \sqrt{\alpha_{A} \beta_{B}} \leq 4 \alpha_{A} \beta_{B}-\alpha_{B} \beta_{A}$, it can be verified that $\alpha_{A} A_{2 i}^{2} \geq \frac{1}{4 \alpha_{A}} \alpha_{i}^{2} \geq \beta_{B} B_{2 i}^{2} \geq \frac{1}{4 \beta_{B}} \beta_{i}^{2}$. (2) When $4 \alpha_{A} \beta_{B}-\beta_{A}^{2} \geq 0$ and $2\left|\beta_{A}\right| \sqrt{\alpha_{A} \beta_{B}} \geq 4 \alpha_{A} \beta_{B}-\alpha_{B} \beta_{A}$, it can be verified that $\alpha_{A} A_{2 i}^{2} \geq$ $\beta_{B} B_{2 i}^{2} \geq \frac{1}{4 \alpha_{A}} \alpha_{i}^{2} \geq \frac{1}{4 \beta_{B}} \beta_{i}^{2}$. (3) When $4 \alpha_{A} \beta_{B}-\beta_{A}^{2} \leq 0$ it can be verified that $\beta_{B} B_{2 i}^{2} \geq \alpha_{A} A_{2 i}^{2} \geq$ $\frac{1}{4 \alpha_{A}} \alpha_{i}^{2} \geq \frac{1}{4 \beta_{B}} \beta_{i}^{2}$. (2) and (3) leads to the second result in the proposition.

\subsection{Proof of Proposition 3}

First, we need to check the conditions in Proposition 2. It is straightforward to verify that $4 \alpha_{A} \beta_{B}-\beta_{A}^{2} \geq 0 \Leftrightarrow 4 \frac{q_{A}}{q_{B}} \alpha_{A}^{2}-\alpha_{A}^{2} \geq 0$. Next, $2\left|\beta_{A}\right| \sqrt{\alpha_{A} \beta_{B}} \leq 4 \alpha_{A} \beta_{B}-\alpha_{B} \beta_{A} \Leftrightarrow 2 \alpha_{A}^{2} \sqrt{\frac{q_{A}}{q_{B}}} \leq$

$4 \frac{q_{A}}{q_{B}} \alpha_{A}^{2}-\alpha_{A}^{2}$. By $2 \sqrt{\frac{q_{A}}{q_{B}}} \leq 3 \frac{q_{A}}{q_{B}} \Leftrightarrow \frac{2}{3}<1 \leq \sqrt{\frac{q_{A}}{q_{B}}}$, it follows that $2 \alpha_{A}^{2} \sqrt{\frac{q_{A}}{q_{B}}} \leq 3 \frac{q_{A}}{q_{B}} \alpha_{A}^{2}<4 \frac{q_{A}}{q_{B}} \alpha_{A}^{2}-\alpha_{A}^{2}$.

By substituting exogenous parameters, the critical values of revenues can be derived as follows

$$
\begin{aligned}
\frac{\alpha_{2}^{2}}{4 \alpha_{A}} & =\frac{\left(q_{A}-q_{B}\right)}{4} \\
\frac{\beta_{1}^{2}}{4 \beta_{B}} & =\frac{\left(q_{A}-q_{B}\right)}{4 q_{A} q_{B}}
\end{aligned}
$$




$$
\begin{aligned}
& \alpha_{A} A_{21}^{2}=\alpha_{A}\left(\frac{\alpha_{B} \beta_{1}}{4 \alpha_{A} \beta_{B}-\alpha_{B} \beta_{A}}\right)^{2}=\frac{\left(q_{A}-q_{B}\right)}{\left(4 q_{A}-q_{B}\right)^{2}} \\
& \beta_{B} B_{21}^{2}=\beta_{B}\left(\frac{2 \alpha_{A} \beta_{1}}{4 \alpha_{A} \beta_{B}-\alpha_{B} \beta_{A}}\right)^{2}=\frac{4\left(q_{A}-q_{B}\right) q_{A}}{\left(4 q_{A}-q_{B}\right)^{2} q_{B}} \\
& \alpha_{A} A_{22}^{2}=\alpha_{A}\left(\frac{2 \beta_{B} \alpha_{i}}{4 \alpha_{A} \beta_{B}-\alpha_{B} \beta_{A}}\right)^{2}=\frac{4\left(q_{A}-q_{B}\right) q_{A}^{2}}{\left(4 q_{A}-q_{B}\right)^{2}} \\
& \beta_{B} B_{22}^{2}=\beta_{B}\left(\frac{\beta_{A} \alpha_{i}}{4 \alpha_{A} \beta_{B}-\alpha_{B} \beta_{A}}\right)^{2}=\frac{\left(q_{A}-q_{B}\right) q_{B} q_{A}}{\left(4 q_{A}-q_{B}\right)^{2}}
\end{aligned}
$$

\section{References}

Acquisti, Alessandro, Hal Varian. 2005. Conditioning prices on purchase history. Marketing Science 24(3) 367-381.

Chen, Yuxin, Ganesh Iyer. 2002. Consumer addressability and customized pricing. Marketing Science $21(2)$ 197-208.

Chen, Yuxin, Chakravarthi Narasimhan, Z. John Zhang. 2001. Individual marketing with imperfect targetability. Marketing Science 20(1) 23-41.

Choudhary, Vidyanand, Anindya Ghose, Tridas Mukhopadhyay, Uday Rajan. 2005. Personalized pricing and quality differentiation. Management Science 51(7) 1120-1130.

Corts, Kenneth. 1998. Third-degree price discrimination in oligopoly:all-out competition and strategic commitment. The Rand Journal of Economics 29(2) 306-323.

Gal-Or, Esther. 1985. Information sharing in oligopoly. Econometrica 53(2) 329-343.

Gal-Or, Esther. 1986. Information transmission-cournot and bertrand equilibria. Review of Economic Studies 53(172) 85.

Gal-Or, Esther, Anindya Ghose. 2005. The economic incentives for sharing security information. Information Systems Research 16(2) 186-208.

Lee, Hau L., Kut C. So, Christopher S. Tang. 2000. The value of information sharing in a two-level supply chain. Management Science 46(5) 626.

Li, Lode. 2002. Information sharing in a supply chain with horizontal competition. Management Science 48(9) 1196-1212. 
Liu, Yunchuan, Z. John Zhang. 2006. The benefits of personalized pricing in a channel. Marketing Science 25(1) 97-105.

Raith, Michael. 1996. A general model of information sharing in oligopoly. Journal of Economic Theory 71(1) 260-288.

Raju, Jagmohan S., Abhik Roy. 2000. Market information and firm performance. Management Science 46(8) 1075.

Sakai, Yasuhiro. 1985. The value of information in a simple duopoly model. Journal of Economic Theory 36(1) 36.

Sasaki, Dan. 2001. The value of information in oligopoly with demand uncertainty. Journal of Economics 73(1) 1-23.

Schmalensee, Richard. 1981. Output and welfare implications of monopolistic third-degree price discrimination. American Economic Review 71(1) 242-247.

Shaffer, Greg, Z. John Zhang. 1995. Competitive coupon targeting. Marketing Science 14(4) 395-416.

Shaffer, Greg, Z. John Zhang. 2000. Pay to switch or pay to stay: Preference-based price discrimination in markets with switching costs. Journal of Economics and Management Strategy 9(3) 397-424.

Shaffer, Greg, Z. John Zhang. 2002. Competitive one-to-one promotions. Management Science 48(9) $1143-1160$.

Shapiro, Carl. 1986. Exchange of cost information in oligopoly. Review of Economic Studies 53(174) 433.

Thisse, Jacques-Francois, Xavier Vives. 1989. On the strategic choice of spatial price policy. American Economic Review 78(1) 122-138.

Varian, Hal. 1992. Microeconomic Analysis. 3rd ed. WW Norton, New York NY.

Varian, Hal R. 1985. Price discrimination and social welfare. American Economic Review 75(4) 870-875.

Villas-Boas, Miguel. 1999. Dynamic competition with customer recognition. The Rand Journal of Economics 30(4) 604-631.

Villas-Boas, Miguel. 2004. Price cycles in markets with customer recognition. The Rand Journal of Economics 35(3) 486-501.

Vives, Xavier. 1984. Duopoly information equilibrium: Cournot and bertrand. Journal of Economic Theory 34(1) 71 .

Ziv, Amir. 1993. Information sharing in oligopoly: The truth-telling problem. RAND Journal of Economics 24(3) 455-465. 